\title{
Tests of selected anti-vibration gloves available on the Polish market
}

\author{
Piotr Kowalski ${ }^{1}$, Jacek Zając ${ }^{2}$ \\ Central Institute for Labour Protection - National Research Institute, Warsaw, Poland \\ ${ }^{1}$ Corresponding author \\ E-mail: ${ }^{1}$ pikow@ciop.pl, ${ }^{2}$ jazaj@ciop.pl \\ Received 14 December 2021; accepted 27 December 2021 \\ DOI https://doi.org/10.21595/vp.2021.22343
}

Check for updates

Copyright (C) 2022 Piotr Kowalski, et al. This is an open access article distributed under the Creative Commons Attribution License, which permits unrestricted use, distribution, and reproduction in any medium, provided the original work is properly cited.

\begin{abstract}
The authors of the paper conducted tests of anti-vibration gloves available on the market (17 types) in accordance with the methodology of the EN ISO 10819: 2013 standard. The main purpose of these studies was to verify the data provided by manufacturers in product specifications and user manuals. Based on the determined values of the vibration transmissibility coefficients, it was found that, despite the certificates held by all the tested gloves, as many as 6 types do not meet the minimum requirements for anti-vibration gloves, and two types achieve properties on the verge of meeting these requirements. Two types of gloves (despite the certificate) did not show any significant vibration damping in the whole tested frequency range. The obtained test results indicate the need to verify the anti-vibration properties of the available certified gloves from unverified suppliers. It also seems necessary to assess the effectiveness of protection of antivibration gloves in the conditions of their actual use.
\end{abstract}

Keywords: vibration, gloves, transmissibility.

\section{Introduction}

Despite the fact that the tests of anti-vibration gloves have been conducted for many years, the rapidly changing product range on the market and the provision of only very limited data by the manufacturers makes it necessary to update and extend the information available to employers (including health and safety services) in such a way that they can correctly their use. This need is also confirmed by the fact that among the many types of tested gloves, only a few new designs meet the minimum requirements set out in EN ISO 10819: 2013 and are certified. At the same time, there are many new models of non-certified gloves on the market that are sold as anti-vibration gloves. Some of the gloves on offer have certificates from many years ago. The introduction of a new test method in 2013 meant that the parameters specified by the manufacturers of anti-vibration gloves, determined before that date, cannot be directly compared with the parameters of the gloves tested with the current method.

Employers are required by law to implement technical measures aimed at limiting exposure to mechanical vibrations (including Directive 2002/44/EC). Currently, with regard to anti-vibration gloves, with the available data on their anti-vibration properties, it is very difficult or impossible to implement. Incorrect selection of gloves in many cases results in an unknowing increase in worker exposure (vibration amplification by gloves).

The authors of the article conducted tests of anti-vibration gloves available on the market (17 types) in accordance with the methodology of the EN ISO 10819: 2013 standard. The main purpose of these studies was to verify the data provided by manufacturers in product specifications and user manuals. In addition, the test results were used to obtain and/or supplement the data necessary for the selection of gloves for the tools/working conditions.

\section{Aim of the study}

The main purpose of these studies was to verify the data provided by manufacturers in product specifications and user manuals. In addition, the test results were used to obtain and/or supplement 
the data necessary for the selection of gloves for the hand tools/working conditions. Presented study is a part of larger project on selection of gloves for the hand tools.

\section{Test objects}

After recognizing the availability of anti-vibration gloves on the market, their initial selection was carried out based on the CE certificates declared by the manufacturers. Each of the selected types of gloves had a marking and information about the compliance of the product with the requirements of EN ISO 10819 Mechanical Vibration and shock - Hand-arm vibration Measurement and evaluation of the vibration transmissibility of gloves at the palm of the hand. Measurement and evaluation of the coefficient of transmissibility of vibrations by gloves to the operator's hand. The 17 types of gloves were selected for the tests.

Due to the interests of manufacturers, the article uses the numbers assigned to them for testing purposes instead of the names of the gloves. There are also illustrative photos that do not allow for unequivocal identification of the presented products.
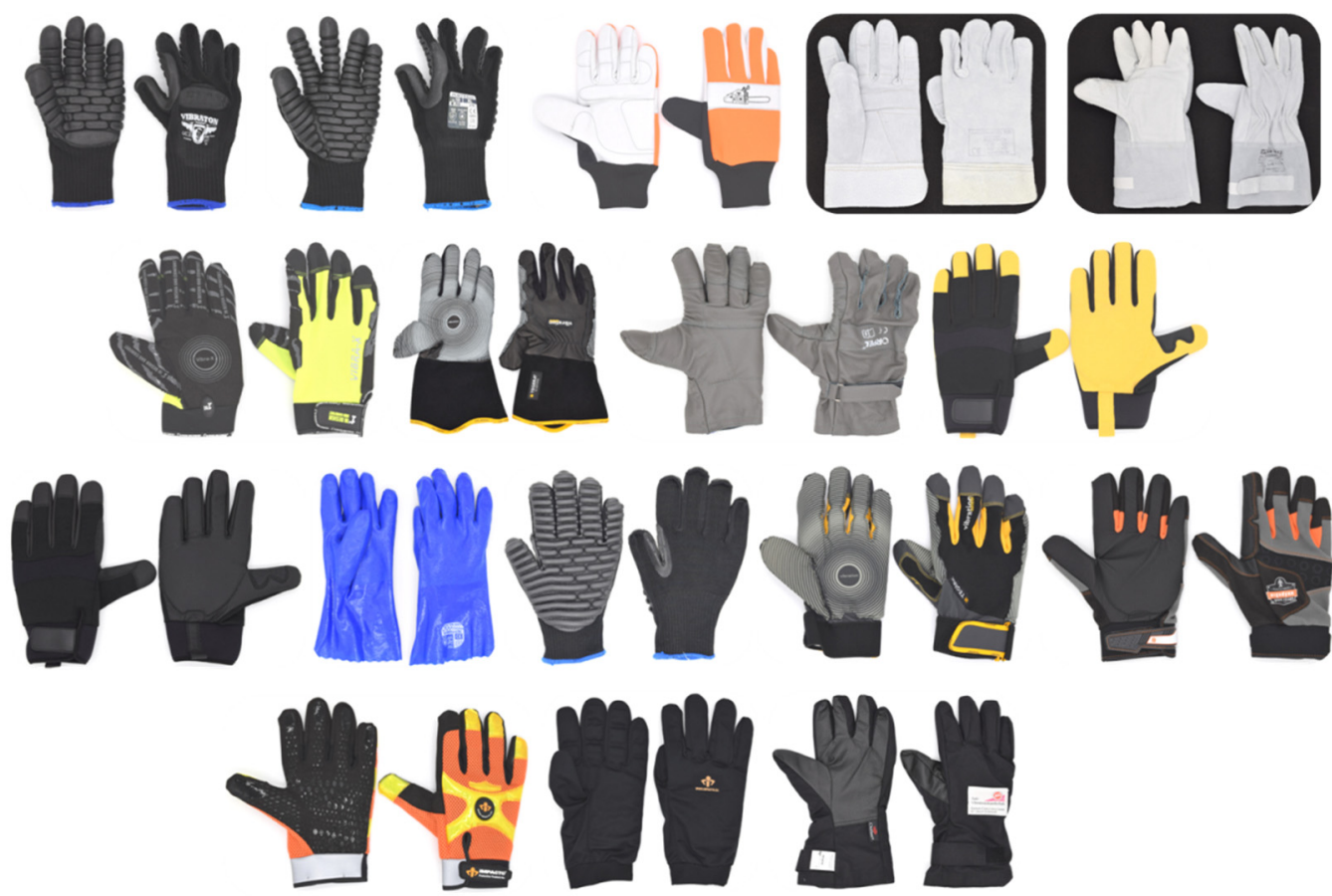

Fig. 1. Tested gloves

\section{Methodology}

A laboratory vibration generation system with a control system was used for the tests, the diagram of which is shown in Fig. 2. Laboratory tests of anti-vibration gloves were carried out on the basis of the method described in EN ISO 10819: 2013.

During the tests, the operator's hand in the tested glove is excited to vibrate by a band-filtered noise signal with a frequency range of 25 to $1250 \mathrm{~Hz}$. The feed and grip forces exerted by the operators on the test handle are kept constant at $50 \mathrm{~N}$ for the feed force and $30 \mathrm{~N}$ for the grip force, respectively.

The amplitude-frequency characteristic of the excitation signal includes 2 parts: from $25 \mathrm{~Hz}$ to $250 \mathrm{~Hz}$ with a constant vibration velocity and from 315 to $1650 \mathrm{~Hz}$ with a falling edge. The total value of the uncorrected vibration acceleration of the test signal is $90.4 \mathrm{~m} / \mathrm{s}^{2}$, and the total 
value of the vibration acceleration corrected by the $W_{h}$ (in accordance with PN EN 8041) characteristic is $4.92 \mathrm{~m} / \mathrm{s}^{2}$.

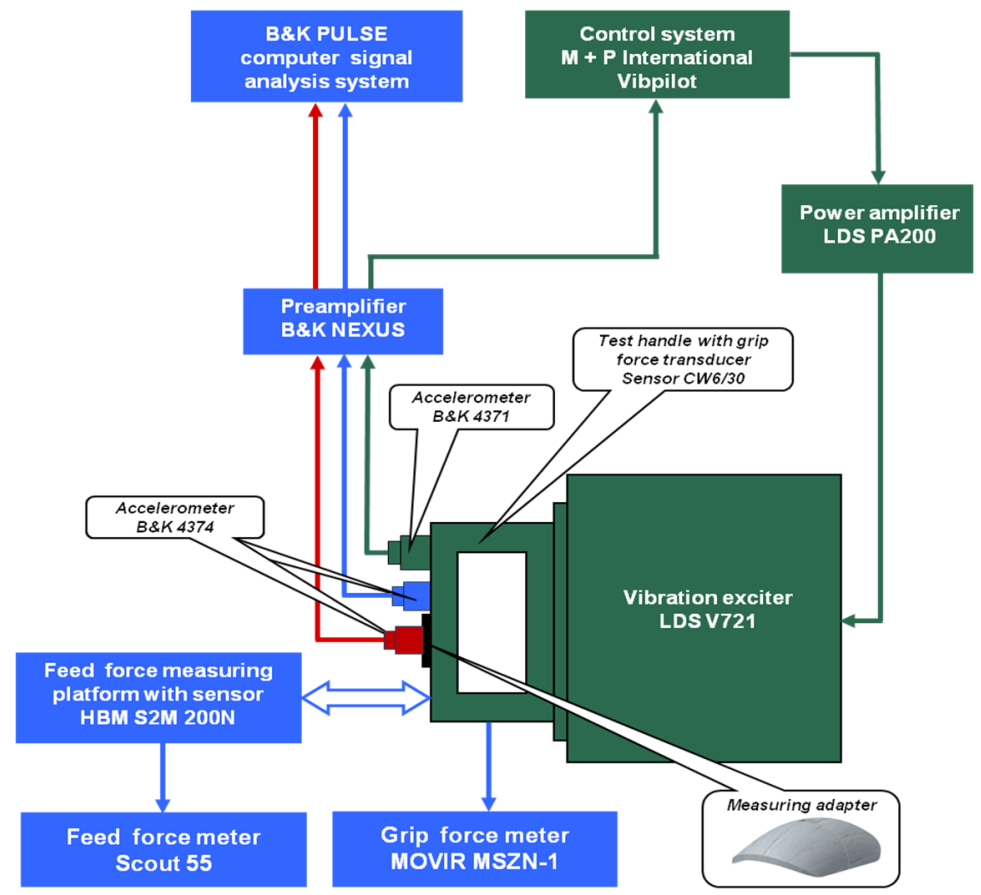

Fig. 2. Diagram of the test stand for testing anti-vibration gloves

The main result of the research are the vibration transmissibility coefficients determined for two frequency ranges of one-third octave bands:

1) $\bar{T}_{(M)}$ factor in the frequency range $\Delta f M: 25 \mathrm{~Hz}-200 \mathrm{~Hz}$ and

2) $\bar{T}_{(H)}$ coefficient in the frequency range $\Delta f H: 200 \mathrm{~Hz}-1250 \mathrm{~Hz}$.

Based on the values of the coefficients $\bar{T}_{(M)}$ and $\bar{T}_{(H)}$ the anti-vibration properties of the gloves are assessed. The coefficients are determined based on the measurements of uncorrected vibration acceleration values in one-third octave bands simultaneously on the test handle and on the measuring adapter (placed directly on the operator's hand). For each glove, 3 separate measurements of vibration acceleration are made in third octave bands for each of the five operators (15 measurements in total).

Uncorrected and corrected by the $W_{h}$ characteristic, the vibration transmissibility coefficients are calculated on the basis of the results of vibration acceleration measurements on the test handle and on the operator's hand. The calculations take into account the characteristics of the vibration transmissibility of the measuring adapter (Fig. 2).

Gloves are considered anti-vibration when they meet both of the following criteria $\bar{T}_{(M)} \leq 0.9$ and $\bar{T}_{(H)} \leq 0.6$.

Since the vibration acceleration characteristics recorded on hand tools in one-third octave bands do not allow for precise determination of the dominant frequencies as well as the resonance frequencies, for the purpose of selecting gloves for tools, the presented methodology was extended by measuring narrow-band vibration transmissibility characteristics and determining the following coefficients:

1) $\bar{T}_{f[M]}$ - narrowband vibration transmissibility coefficient in the frequency range $\Delta f_{M}$ $(22-225 \mathrm{~Hz})$, 
2) $\bar{T}_{f[H]}$ - narrowband vibration transmissibility coefficient in the frequency range $\Delta f_{H}$ $(178-1403 \mathrm{~Hz})$,

3) $\bar{T}_{f}$ - narrowband vibration transmissibility coefficient in the frequency range $\Delta f$ $(1-1600 \mathrm{~Hz})^{*}$. (*As the test signal is defined in the frequency range $22-1403 \mathrm{~Hz}$, tests outside this range should be considered approximate.)

Coefficients: $\bar{T}_{f[M]}, \bar{T}_{f[H]}, \bar{T}_{f}$ are calculated in the same way as in the case of tests with the use of vibration acceleration characteristics in one third octave bands.

The calculations were performed using the Matlab computing environment and the Excel spreadsheet.

Five testers (operators) participated in the tests of each type of glove. Their height was in the range of $167-182 \mathrm{~cm}$ and weight in the range of $63-84 \mathrm{~kg}$. Three tests were performed with the participation of each of the testers; a total of 15 tests for one type of glove.

\section{Laboratory test results of gloves}

Table 1 shows the average values of the vibration transmissibility coefficients for all tested gloves. The green color is used to distinguish the values characterizing gloves with the best (among the tested) anti-vibration properties, and the red color shows the values characterizing the gloves with the worst anti-vibration properties (vibration amplification).

Table 1. Averaged vibration transmissibility coefficients for gloves

\begin{tabular}{|c|c|c|c|c|c|}
\hline No. of glove & $\bar{T}_{[M]}$ & $\bar{T}_{f[M]}$ & $\bar{T}_{[H]}$ & $\bar{T}_{f[H]}$ & $\bar{T}_{f}$ \\
\hline 1 & 0,866 & 0.883 & 0,888 & 0.918 & 0.895 \\
\hline 2 & 0,848 & 0.855 & 0,849 & 0.854 & 0.854 \\
\hline 3 & 0,833 & 0.840 & 0,820 & 0.826 & 0.835 \\
\hline 4 & 0,934 & 0.942 & 1,041 & 1.047 & 0.982 \\
\hline 5 & 0,931 & 0.938 & 1,024 & 1.030 & 0.974 \\
\hline 6 & 0,725 & 0.731 & 0,609 & 0.614 & 0.691 \\
\hline 7 & 0,691 & 0.696 & 0,494 & 0.498 & 0.634 \\
\hline 8 & 0,673 & 0.678 & 0,473 & 0.477 & 0.617 \\
\hline 9 & 0.752 & 0.750 & 0.561 & 0.562 & 0.692 \\
\hline 10 & 0.729 & 0.731 & 0.555 & 0.554 & 0.674 \\
\hline 11 & 0.696 & 0.701 & 0.488 & 0.493 & 0.635 \\
\hline 12 & 0,684 & 0.685 & 0,542 & 0.543 & 0.636 \\
\hline 13 & 0,747 & 0.738 & 0,597 & 0.604 & 0.690 \\
\hline 14 & 0,718 & 0,719 & 0,536 & 0.538 & 0.660 \\
\hline 15 & 0,705 & 0,707 & 0,546 & 0.548 & 0.654 \\
\hline 16 & 0,721 & 0,722 & 0,618 & 0.619 & 0.687 \\
\hline 17 & 0,844 & 0,846 & 0,830 & 0.832 & 0.841 \\
\hline
\end{tabular}

As expected, the obtained values of the vibration transmissibility coefficients obtained on the basis of the third octave $\left(\bar{T}_{(M)}\right.$ and $\left.\bar{T}_{(H)}\right)$ and narrowband $\left(\bar{T}_{f[M]}, \bar{T}_{f[H]}\right.$ characteristics) do not differ by more than $2 \%$. They can therefore be used interchangeably. The coefficient $\bar{T}_{f}$ can be used as a single-number index for a quick, rough assessment of the anti-vibration properties of gloves.

Based on the values of vibration transmissibility coefficients obtained by the tested gloves, it can be stated that out of 17 types of gloves with EU-type examination certificates (requirements of the Regulation of the European Parliament and of the Council (EU) 2016/425 of March 9, 2016), as many as 6 (No. 2, 3, 4, 5, 17) does not meet the minimum requirements for anti-vibration gloves specified in the EN ISO 10819: 2013 standard, and two types (No. 6 and 16) achieve properties on the verge of meeting these requirements.

The determined values of standard deviations: $s_{T(M)}, s_{T(H)}, s_{T f(M)}, s_{T f(H)}, s_{T f}(<0.073)$ and coefficients of variation: $C_{V, T(M)}, C_{V, T(H)}, C_{V, T f(M)}, C_{V, T f(H)}, C_{V, T f}(<0.147)$, both for calculations 
based on third octave characteristics and obtained from narrowband characteristics, indicate good reproducibility of the measurement results. An unambiguous assessment of these values is difficult, because the research method takes into account the participation of people with different weight and body build, which means that the measurement results obtained during tests with the participation of individual testers differ in principle.

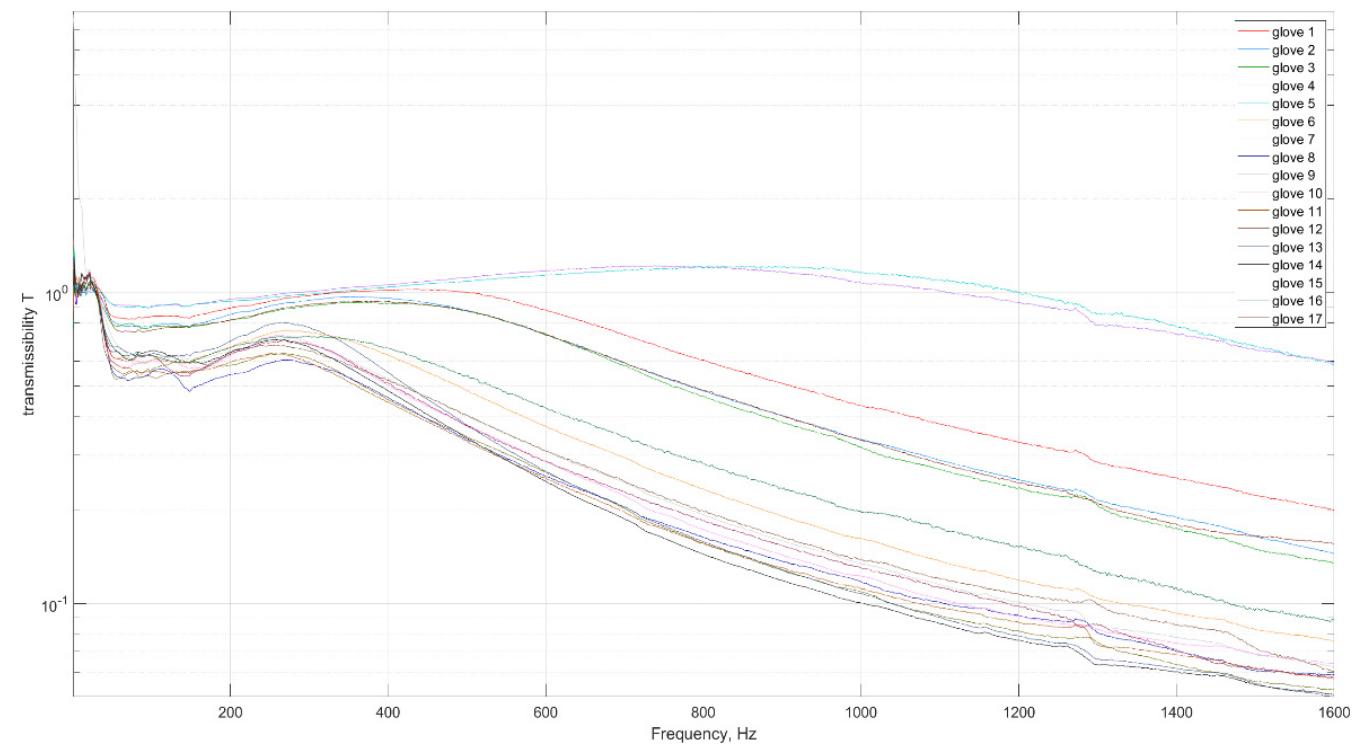

Fig. 3. Averaged narrowband vibration transmissibility characteristics of 17 tested gloves

Fig. 3 presents a summary of averaged, narrowband vibration transmissibility characteristics determined for all 17 tested types of gloves. Analyzing the presented characteristics of the transmissibility of vibrations, it was found that:

1) in all vibration transmissibility characteristics, two frequency ranges can be distinguished with two local maxima; the first of them is related to the strengthening of vibrations by the gloves, while the second one is to increase the vibrations only for 3 types of gloves (No. 1, 4, 5),

2 ) in the frequency range 1-35 Hz, all tested gloves slightly (except for gloves No. 9 for which there was a strong strengthening) amplify vibrations,

3 ) outside the frequency range 1-35 Hz, gloves No. 1, 4, 5 also amplify vibrations, while in the widest frequency range $(300-1200 \mathrm{~Hz})$ gloves no. 4 and 5 .

4) apart from gloves No. 1, 2, 3, 4, 5, 17, other gloves show good vibration damping in the frequency range $300-1600 \mathrm{~Hz}$,

5) gloves No. 4 and 5 do not show any significant vibration damping in the whole tested frequency range,

6) gloves No. 1, 2, 3, 17 show the damping of vibrations only in the range of higher frequencies, i.e. approx. $700-1600 \mathrm{~Hz}$.

7) The highest vibration damping in the frequency range $35-300 \mathrm{~Hz}$ is shown by gloves No. 8 .

Comparing the characteristics of gloves with a very similar (almost identical) design: No. 1, 2 and 12, it was observed that gloves No. 1 and 2 had significantly worse anti-vibration properties than gloves No. 12. This is also confirmed by the calculated values of vibration transmissibility coefficients (Table 1) which shows that gloves No. 1 and 2 should not be certified for antivibration gloves $\left(\bar{T}_{(H)}>0.6\right)$. 


\section{Conclusions}

Based on the results of the tests, the following conclusions were drawn:

1) The obtained values of the vibration transmissibility coefficients obtained on the basis of the third octave and narrowband characteristics do not differ by more than $2 \%$ and can be used interchangeably.

2) On the basis of the determined values of the vibration transmissibility coefficients, it was found that despite the certificates held by all tested gloves (17 types), as many as 6 types do not meet the minimum requirements for anti-vibration gloves, and 2 types achieve properties on the verge of meeting these requirements.

3) By analyzing the frequency characteristics of the vibration transmissibility through the gloves, it was found that in the frequency range 1-35 Hz all the tested gloves slightly (except for gloves No. 9 for which there was a strong amplification) strengthen the vibrations; apart from gloves with vibration amplification in the range of $35-1600 \mathrm{~Hz}$, the remaining gloves show good vibration damping in the frequency range 300-1600 Hz, 2 types of gloves (despite the certificate) do not show significant vibration damping in the whole tested frequency range.

4) It was found that three types of gloves with a very similar (almost identical) design have diametrically different anti-vibration properties. Among them, two types should not be certified for anti-vibration gloves.

The obtained test results indicate the need to verify the anti-vibration properties of the available certified gloves from unverified suppliers. It also seems necessary to assess the effectiveness of protection of anti-vibration gloves in the conditions of their actual use.

The operation of consumer protection mechanisms in relation to the anti-vibration gloves market has proved to be insufficient.

\section{Acknowledgements}

This paper has been based on the results of a research task carried out within the fifth stage of the National Programme "Improvement of Safety and Working Conditions" partly supported in 2021-2022 - within the scope of state services - by the Minister Responsible for Labour (task No. 3.SP.05 entitled "Development of Requirements for the Correct Selection of Anti-Vibration Gloves"). The Central Institute for Labour Protection - National Research Institute is the Programme's main co-ordinator.

\section{References}

[1] Koton J. and Kowalski P., "Selection of personal protective equipment, Chap," Mechanical vibrations Warszawa, 2007.

[2] P. Kowalski, "Examining the effectiveness of anti-vibration gloves with a neural network," International Journal of Occupational Safety and Ergonomics, Vol. 17, No. 3, pp. 241-247, Jan. 2011, https://doi.org/10.1080/10803548.2011.11076891

[3] Majchrzycka K. and Pościk A., "Selection of personal protection measures," in Mechanical Vibrations, 2007, pp. 215-227.

[4] Zając J., Kowalski P., and Rejman M., "Test method for anti-vibration gloves according to the standard EN ISO 10819:2013," Mechanical Review, Vol. 1, No. 3, pp. 46-50, Mar. 2018, https://doi.org/10.15199/148.2018.3.5

[5] "Directive 2002/44/WE of the European Parliament and of the Council of 25 June 2002 on The Minimum Health and Safety Requirements Regarding the Exposure of Workers to the Risks Arising from Physical Agents (Vibration)," Sixteenth Individual Directive Within the Meaning of Article 16(1) of Directive 89/391/EEC, 2002.

[6] "ISO 10819:2013-12 Mechanical Vibration and Shock - Hand-Arm Vibration - Measurement and Evaluation of the Vibration Transmissibility of Gloves at the Palm of the Hand," 2013. 